\title{
EDITORIAL
}

\section{Non-invasive coronary imaging: computed tomography or magnetic resonance imaging?}

\section{R Peebles}

Heart 2003;89:591-594

\begin{abstract}
Non-invasive coronary artery imaging has taken a significant step forward with the advent of multislice computed tomography
\end{abstract}

$\mathrm{N}$ on-invasive coronary imaging has recently seen a rapid expansion of interest, fuelled largely by technical advances in both computed tomography (CT) and cardiac magnetic resonance imaging (CMR). The race is on for these two modalities to provide routine and robust imaging of coronary arteries in clinical practice.

The rate of this progress has inevitably provided a bewildering array of developments, which are hard to stay abreast of. This article will attempt to clarify the fundamental differences between the available techniques, explore their respective capabilities, and discuss future potentials.

\section{ELECTRON BEAM COMPUTED TOMOGRAPHY (EBCT)}

EBCT has been the CT method of choice for imaging the heart for the last 10 years or more. Unlike conventional CT, which uses a rotating $x$ ray tube, EBCT uses a static row of detectors and a moving beam of electrons to produce the $x$ ray photons. The advantage of this is a rapid image acquisition time of 50-100 ms per slice. When combined with ECG triggering this allows imaging of a motion free heart during diastole (usually $80 \%$ of the RR interval). ${ }^{1}$ This technique has been used to quantify coronary calcium burden and more recently to perform contrast enhanced coronary angiography. ${ }^{2}{ }^{3}$ The disadvantages of EBCT are the poor signal-to-noise ratio and increased slice thickness compared with conventional CT, the lack of general availability of EBCT, and the radiation dose compared to CMR.

\section{MULTISLICE COMPUTED TOMOGRAPHY (MSCT)}

Also known as multidetector CT, these scanners are a development from single slice helical (or spiral) CT. A single rotating $x$ ray tube provides a beam of photons that is then received by several rows of detectors (usually four), effectively giving four axial slices for each tube rotation. There are now scanners available that allow eight or 16 slices per rotation. This technique allows much greater coverage than a conventional helical scanner for a given acquisition time and slice thickness. Thus the whole heart can be covered in one breath-hold with a slice thickness of $1 \mathrm{~mm}$.
The temporal resolution is less good than EBCT, being limited by a tube rotation time of $500 \mathrm{~ms}$. If only $180^{\circ}$ of the data is interpolated this allows a slice acquisition time of $125-250 \mathrm{~ms} .{ }^{4}$ This causes significant problems with cardiac movement, particularly at faster heart rates ( $>70$ beats/min), and most investigators give a $\beta$ blocker before the examination.

The advantage over EBCT is improved signalto-noise ratio and spatial resolution ( $1 \mathrm{~mm}$ slice width versus $3 \mathrm{~mm}$ slice width). The disadvantage is increased radiation dose compared with EBCT. Because the heart is imaged throughout the cardiac cycle but only data obtained during diastole are used (retrospective gating), a large volume of obtained data is unused, contributing to the radiation dose but not the image.

\section{CARDIAC MR (CMR)}

The absence of ionising radiation, and lack of need for iodinated contrast medium, makes CMR the theoretical method of choice for non-invasive coronary imaging. The task for CMR is the same as for CT, namely to image as much of the coronary circulation as possible, with maximal spatial and temporal resolution, maximal signal-to-noise ratio, and short imaging time. The development of new sequences has allowed several different strategies for coronary imaging to evolve. Initial coronary studies were performed using multiple two dimensional slices during a breath-hold. ${ }^{5}$ Two dimensional sequences, however, suffer from poor signal-to-noise ratio, problems with misregistration (relating to multiple breath-holds), and place considerable demands on the operator. Three dimensional sequences have now been developed for both breath-hold and free breathing techniques. The latter use a navigator echo to track the diaphragm and retrospectively gate the acquisition for respiratory motion. ${ }^{6}$ Breath-hold three dimensional images using a variety of ultra-fast sequences are now a reality and produce excellent image quality because of the short acquisition times. ${ }^{7}$ Paramagnetic contrast medium may be added to any of these techniques in an attempt to increase contrast to noise ratio and improve stenosis visualisation. $^{5}$

\section{CORONARY LUMEN IMAGING}

In an article by Leber and colleagues in this issue of Heart, the use of contrast enhanced, multislice

Abbreviations: $C T$, computed tomography; $E B C T$, electron beam computed tomography; CMR, cardiac magnetic resonance imaging; IVUS, intravascular ultrasound; MSCT, multislice computed tomography 
Table 1 Summary of trials comparing CMR, EBCT, and MSCT with invasive coronary angiography

\begin{tabular}{|c|c|c|c|c|c|c|}
\hline Modality & Author & $\begin{array}{l}\text { Patient } \\
\text { numbers }\end{array}$ & Sensitivity (\%) & Specificity (\%) & $\begin{array}{l}\text { Coronary segments } \\
\text { not assessable (\%) }\end{array}$ & $\begin{array}{l}\text { Patients excluded } \\
\text { because of poor image } \\
\text { quality }\end{array}$ \\
\hline \multirow[t]{6}{*}{ Cardiac MR } & Post et $\left.a\right|^{b}$ & 20 & 38 & 95 & 4 & \\
\hline & Van Geuns et aP & 38 & 68 & 97 & 31 & \\
\hline & Sandstede et al & 30 & 81 & 89 & & 7 \\
\hline & Regenfus et a $1^{10}$ & 50 & 94 & 57 & 33 & \\
\hline & Kessler et $a l^{11}$ & 73 & 65 & 88 & 48 & 8 \\
\hline & Kim et $a l^{12}$ & 109 & 93 & 42 & 16 & 6 \\
\hline \multirow[t]{4}{*}{ Electron beam CT } & Leber et $a l^{13}$ & 93 & 78 & 93 & 24 & 6 \\
\hline & Achenbach et $a l^{14}$ & 125 & 92 & 94 & 25 & \\
\hline & Schmermund et $a l^{15}$ & 28 & 82 & 88 & 28 & \\
\hline & Reddy et al ${ }^{16}$ & 23 & 88 & 79 & 10 & \\
\hline \multirow[t]{3}{*}{ Multislice CT } & Nieman et al $1^{17}$ & 35 & 81 & 97 & 26 & \\
\hline & Knez et $a l^{18}$ & 44 & 78 & 98 & 6 & 1 \\
\hline & Giesler et a ${ }^{19}$ & 100 & 91 & 89 & 29 & \\
\hline
\end{tabular}

CT coronary angiography is compared with electron beam $\mathrm{CT}^{8}{ }^{8}$ One hundred and one patients were enrolled to the electron beam group and 91 patients to the MSCT group. Both groups were comparable with the usual CT exclusion criteria applied, notably atrial fibrillation, renal disease, and iodinated contrast allergy. The EBCT and MSCT techniques used are well described and were optimised in the MSCT group with oral or intravenous $\beta$ blockade before investigation if the patients had a heart rate over 70 beats/min. Selective coronary angiography was used as the gold standard in both groups. Significant coronary stenoses $(>50 \%)$ were identified with a sensitivity of $77 \%$ and $82 \%$ using EBCT and MSCT, respectively. Specificity was also comparable, $93 \%$ for EBCT and $96 \%$ for MSCT. There were significant differences between EBCT and MSCT in two areas; contrast-to-noise ratio and number of assessable coronary segments, with MSCT performing marginally better in each.

While these results may appear impressive they have only been obtained by exclusion of coronary segments that could not be assessed by $\mathrm{CT}$, and only attempting to examine the proximal two thirds of the coronary tree. This practice is common to all of the studies looking at non-invasive coronary imaging and, although understandable, does tend to give a misleading accuracy to the results. In the present study only $76 \%$ of EBCT and $82 \%$ of MSCT segments could be assessed, the remainder being excluded largely because of motion artefact or poor contrast-to-noise ratio. This highlights the main problem of non-invasive coronary imaging for all modalitiesthe difficulty of reliably visualising all the coronary segments, particularly in the fast moving right coronary and circumflex vessels. Previous CMR, EBCT, and MSCT studies ${ }^{679-19}$ demonstrated similar results, with between $10-40 \%$ of coronary segments not assessable (table 1). As well as the problem of coronary motion, other factors such as heavy coronary calcification and coronary stents limit visualisation of the coronary lumen on both CT and CMR.

A further cause for concern with CT techniques, which is rightly raised by the authors in this issue, ${ }^{8}$ is the relatively high radiation doses. EBCT delivers an average dose of $2.7 \mathrm{mSv}$ and MSCT a dose of $8.2 \mathrm{mSv}$; this compares with a conventional angiographic dose of approximately 3-9 mSv. While new technical advances, such as ECG modulated beam current techniques, should reduce the MSCT dose it will continue to be a significant disadvantage of CT compared to CMR.

On a more positive note it is clear that MSCT can compete favourably with EBCT in coronary luminal imaging. Comparison of the results in table 1 indicates very similar sensitivities and specificities between the two techniques, with an equivalent number of segments not imaged. MSCT can be expected to improve significantly in the near future with the introduction of ever larger numbers of detector rows (eight and 16). This will increase the spatial resolution in the $\mathrm{z}$ axis as well as decrease scan times allowing better coverage of the coronary circulation. A reduction in gantry rotation time may also allow improved temporal resolution and reduced motion artefact. The first clinical trials from these scanners are awaited with interest.

Coronary magnetic resonance angiography shows more variable results (table 1). This is in part due to the variety of different techniques used. The most recent trial of Kim and colleagues was a multicentre study using a free breathing three dimensional technique. ${ }^{12}$ In 109 patients this group found a very high sensitivity (100\%) and negative predictive value $(100 \%)$ for patients with left main stem or three vessel disease. The conclusion was that CMR would be useful as a screening technique for three vessel and left mainstem disease-for example, in patients with dilated cardiomyopathy. The overall accuracy was $72 \%$, with a positive predictive value of $70 \%$, having excluded $16 \%$ of coronary segments (unassessable). Considering the variable experience of the centres involved these results reflect a promising advance in the robustness of CMR in the clinical setting.

The problems for CMR are similar to those of CT. Patients need to be in sinus rhythm and even then a high number of examinations produce poor quality images that are nondiagnostic. In the study by Kessler and colleagues, eight of 73 patients were excluded because of unsatisfactory image quality, and even then only 236 of 455 segments could be evaluated for stenoses. ${ }^{11}$ Although results have and will continue to improve, this lack of robustness remains a problem.

\section{CORONARY ARTERY WALL AND PLAQUE IMAGING}

Both CT and MRI techniques have made strides in imaging the coronary artery wall in an attempt to identify vulnerable plaques. EBCT is reliably able to quantify coronary calcium indicating atherosclerosis burden. ${ }^{20}$ More recently MSCT has also demonstrated this ability. ${ }^{21}$ The use of calcium scores is controversial, however, as a high score has not yet been proven to be additive to conventional risk factor stratification in terms of prognosis. ${ }^{22}$ In addition a low calcium score does not exclude the vulnerable lipid rich plaques that are at most risk of rupture. Hopefully large multicentre trials will allow more critical appraisal of the prognostic use of coronary calcium scoring. ${ }^{23}$

MSCT has been shown to identify soft, intermediate, and calcified plaques in humans, albeit in a small cohort of 15 patients. ${ }^{24}$ This study compared MSCT with intravascular 
ultrasound (IVUS) and invasive angiography in selected lesions. The density of the plaque on CT correlated significantly with the IVUS classification of plaque morphology. Further larger studies are needed, but this is a promising development in our ability to identify vulnerable plaque noninvasively, and subsequently stratify risk in individual patients.

There are a number of studies that address the feasibility of plaque imaging with MRI in both humans and animals. These have shown significant correlation between arterial wall thickness and atherosclerotic burden in human coronaries and aortas, as well as pig coronaries. ${ }^{25-27}$ Most recently a study of rabbits allowed longitudinal follow up of aortic plaque burden/composition with MRI. ${ }^{28}$ There was progression of both plaque volume and lipid component in rabbits on an atherogenic diet, while there was regression when on a normal diet. These findings correlated well with histological specimens. The authors, quite reasonably, suggest that there is the potential to use MRI to document changes in human atheroslerotic plaque burden and composition in response to therapeutic interventions such as lipid lowering agents. Time will tell if similar results can be achieved in human coronary arteries, but it does seem likely that with further improvements in MR techniques routine plaque imaging will be achieved.

\section{CORONARY FLOW}

The ability to measure coronary flow and flow reserve has traditionally been limited to interventional techniques such as Doppler flow wires. These allow the functional assessment of coronary artery and coronary artery bypass graft stenoses as an adjunct to interventional procedures. CMR has the ability to measure flow non-invasively using flow mapping sequences. Hundley and colleagues studied 30 patients with phase contrast MR immediately before coronary angiography. ${ }^{29}$ In comparison to computer assisted quantitative angiography MR flow mapping identified $70 \%$ stenoses of the proximal left anterior descending coronary artery with a sensitivity of $100 \%$ and a specificity of $83 \%$. They found a coronary flow reserve of $<1.7$ in all patients with a stenosis of $>70 \%$ following pharmacological stress with adenosine. There was a good correlation between MR flow reserve and Doppler guide wire flow reserve in a subset of 17 patients. Although this was a selected study population, excluding patients with other factors that may have influenced flow reserve (for example, dilated or hypertrophic cardiomyopathy, severe valvar heart disease, previous anterior infarction, or the presence of coronary bypass grafts), the results are encouraging. This is particularly the case because the MR techniques used were not especially advanced and were performed at two institutions with different scanners. Other groups have looked at flow mapping in bypass vein grafts and left internal mammary artery grafts both with and without pharmacological stress. ${ }^{30-32}$ The most recent of these demonstrated excellent correlation between MR and Doppler flow measurements in non-stenotic and stenotic grafts.

\section{CONCLUSION}

If one makes a direct comparison between EBCT, MSCT, and CMR at the present time it would be difficult to choose a winner. EBCT, the most established non-invasive technique for imaging coronary arteries, is likely to be eclipsed by MSCT. This is largely due to the versatility of multislice scanners and their subsequent appeal to radiology departments. In due course all hospitals are likely to have an MSCT at their disposal while EBCT is a tool that will generally be limited to research groups. The MSCT studies published to date have been performed on four slice scanners and the results from faster multislice scanners are awaited with interest. It seems likely that the improved resolution and shorter acquisition times will make the technique more robust and hopefully allow routine coronary lumen imaging. Taken in conjunction with the ability to define plaque composition, this may tip the balance in favour of MSCT over MR in the short to medium term.

Longer term, however, technical advances in MR are likely to improve significantly the resolution and reliability of coronary MR imaging, both of the lumen and vessel wall. If this is the case, and one adds to the equation the versatility of CMR in terms of coronary flow quantification, myocardial perfusion, myocardial viability, and ventricular function studies, a one stop cardiac imaging service may be possible. The absence of ionising radiation and nephrotoxic contrast is also a considerable incentive to use MR.

The true role that non-invasive coronary imaging is likely to take in the future is still unclear. Initially these techniques seem well placed to screen patients with equivocal chest pain, dilated cardiomyopathy, and bypass grafts. As they become increasingly robust this role is likely to expand to include routine diagnostic angiography. Conventional coronary angiography is likely to remain for the foreseeable future not only as a prelude to coronary intervention but also in those patients who remain unsuitable for MR or CT.

\section{ACKNOWLEDGEMENTS}

Thanks to Bruce Ogilvie and Ivan Brown for their comments on the draft of this paper.

\section{REFERENCES}

1 de Feyter PJ, Nieman K, van Ooijen $\mathrm{P}$, et al. Non-invasive coronary artery imaging with electron beam computed tomography and magnetic resonance imaging. Heart 2000;84:442-8.

2 Agatston AS, Janowitz WR, Hildner FJ, et al. Quantification of coronary artery calcium using ultrafast computed tomography. J Am Coll Cardiol 1990; 15:827-32.

3 Rensing BJ, Bongaerts A, van Guens RJ, et al. Intravenous coronary angiography by electron beam computed tomography. Circulation 1998;98:2509-12.

4 Nieman K, Oudkerk $M$, Rensing BJ, et al. Coronary angiography with multi-slice computed tomography. Lancet 2001;357:599-603.

5 Bunce NH, Pennell DJ. Magnetic resonance of the coronary arteries. Eur Radiol 2001;11:721-31.

6 Post JC, van Rossum AC, Hofman MB, et al. Three-dimensional respiratory-gated MR angiography of coronary arteries: comparison with conventional coronary angiography. AJR 1996;166:1399-404.

7 van Geuns RJ, Wielopolski PA, de Bruin HG, et al. MR coronary angiography with breath-hold targeted volumes: preliminary clinical results. Radiology 2000;217:270-7.

8 Leber AW, Knez A, Becker C, et al. Non-invasive intravenous coronary angiography using electron beam tomography and multislice computed tomography. Heart 2003;89:633-9.

9 Sandstede JJW, Pabst T, Beer M, et al. Three-dimensional MR coronary angiography using the navigator technique compared with conventional coronary angiography. AJR 1999;172:135-9.

10 Regenfus M, Ropers D, Achenbach S, et al. Noninvasive detection of coronary artery stenosis using contrast-enhanced three-dimensional breath-hold magnetic resonance coronary angiography. J Am Coll Cardiol 2000;36:44-50.

11 Kessler W, Achenbach S, Moshage W, et al. Usefulness of respiratory gated magnetic resonance coronary angiography in assessing narrowings $>50 \%$ in diameter in native coronary arteries and in aortocoronary bypass conduits. Am J Cardiol 1997;80:989-93.

12 Kim WY, Danias PG, Stuber $M$, et al. Coronary magnetic resonance angiography for the detection of coronary stenoses. N Engl J Med 2001;345:1863-9.

13 Leber AW, Knez A, Mukheriee R, et al. Usefulness of calcium scoring using electron beam computed tomography and non-invasive coronary angiography in patients with suspected coronary artery disease. Am J Cardiol 2001;88:219-23.

14 Achenbach S, Moshage W, Ropers D, et al. Value of electron beam computed tomography for the non-invasive detection of high-grade coronary-artery stenoses and occlusions. N Engl J Med 1998;339:1964-71.

15 Schmermund A, Rensing BJ, Sheedy PF, et al. Intravenous electron-beam computed tomographic coronary angiography for segmental analysis of coronary artery stenoses. J Am Coll Cardiol 1998;31:1547-54.

16 Reddy GP, Chernoff DM, Adams JR, et al. Coronary artery stenoses: assessment with contrast-enhanced electron-beam CT and axial reconstructions. Radiology 1998;208:167-72.

17 Nieman K, Ouderk M, Rensing BJ, et al. Coronary angiography with multi-slice computed tomography. Lancet 2001;357:599-603.

18 Knez A, Becker CR, Leber A, et al. Usefulness of multislice spiral computed tomography angiography for determination of coronary artery stenoses. Am J Cardiol 2001;88:1191-4. 
19 Geisler T, Baum U, Ropers D, et al. Noninvasive visualization of coronary arteries using contrast-enhanced multidetector CT: influence of heart rate on image quality and stenosis detection. AJR 2002;179:911-16

20 Knollmann FD, Bocksch W, Spieselsberger S, et al. Electron beam computed tomography in the assessment of coronary artery disease after heart transplantation. Circulation 2000;101:2078-82.

21 Carr JJ, Danitschek JA, Goff DC, et al. Coronary artery calcium quantification with retrospectively gated helical CT: protocols and techniques. Int J Cardiovasc Imaging 2001;17:213-220.

22 Schmermund A, Erbel R. Unstable coronary plaque and its relation to coronary calcium. Circulation 2001;104:1682-7.

23 O'Rourke RA, Brundage BH, Froelicher VF, et al. American College of Cardiology/American Heart Association expert consensus document on electron-beam computed tomography for the diagnosis and prognosis of coronary artery disease. Circulation 2000;102:126-40.

24 Schroeder S, Kopp AF, Baumbach A, et al. Noninvasive detection and evaluation of atherosclerotic coronary plaques with multislice computed tomography. J Am Coll Cardiol 2001;37:1430-5.

25 Botnar RM, Stuber M, Kissinger KV, et al. Noninvasive coronary vessel wall and plaque imaging with magnetic resonance imaging. Circulation 2000; 102:2582-7
26 Corti $\mathbf{R}$, Fayad ZA, Fuster V, et al. Effects of lipid-lowering by simvastatin on human atherosclerotic lesions. Circulation 2001;104:249-52.

27 Worthley SG, Helft G, Fayad ZA, et al. Cardiac gated breath-hold black blood MRI of the coronary artery wall: an in vivo and ex vivo comparison. Int J Cardiovasc Imaging 2001;17:195-201.

28 Helft G, Worthley SG, Fuster V, et al. Progression and regression of atherosclerotic lesions, monitoring with serial non-invasive magnetic resonance imaging. Circulation 2002;105:993-8.

29 Hundley WG, Hamilton CA, Clarke GD, et al. Visualization and functional assessment of proximal and middle left anterior descending coronary stenoses in humans with magnetic resonance imaging. Circulation 1999:99:3248-54.

30 Langerak SE, Kunz P, Vliegen HW, et al. Improved MR flow mapping in coronary artery bypass grafts during adenosine-induced stress. Radiology 2001;218:540-7.

31 Miller S, Scheule AM, Hahn U, et al. MR angiography and flow quantification of the internal mammary artery graft after minimally invasive direct coronary artery bypass. AJR 1999;172:1365-9.

32 Langerak SE, Kunz P, Vliegen HW, et al. MR flow mapping in coronary artery bypass grafts: a validation study with Doppler flow measurements. Radiology 2002;222:127-35.

\section{IMAGES IN CARDIOLOGY}

\section{Multiple coronary rupture after blunt chest trauma}

A 78 year old woman with a history of hypertension underwent elective hip arthroprosthesis. Three days later, the patient accidentally fell resulting in luxation of the arthroprosthesis, which was reduced under spinal anaesthesia. Severe hypotension occurred soon after. A chest $x$ ray showed massive left pleural effusion and blood tests documented anaemia that required transfusion. ECG showed new onset atrial fibrillation with a mean ventricular rate of 110 beats/min and chronic left bundle branch block. Transthoracic echocardiography performed at bedside, although suboptimal, showed normal left and right ventricles, near normal left ventricular ejection fraction, and approximately $700 \mathrm{ml}$ of pericardial effusion. The patient died suddenly and a postmortem examination was performed.

Necropsy revealed haemopericardium with no signs of heart rupture. A fistula from the anterolateral surface of the left ventricle to the intermediate coronary artery was present. Histology confirmed the rupture of two branches of the intermediate coronary artery, with sharp edges, fibrin stratification, and infiltration of blood into the subepicardial layers adjacent to the two arteries (panels A-D). Multiorgan stasis, centrolobular hepatic necrosis, and bowel infarction were also present.

Blunt thoracic trauma resulting in coronary artery rupture is uncommon. Cases of dissection or thrombosis of a coronary artery after chest trauma have more often been described. In our case, the damage of two neighbouring arteries and the sharp edges of the rupture seen on histology leave no doubt on its traumatic origin.

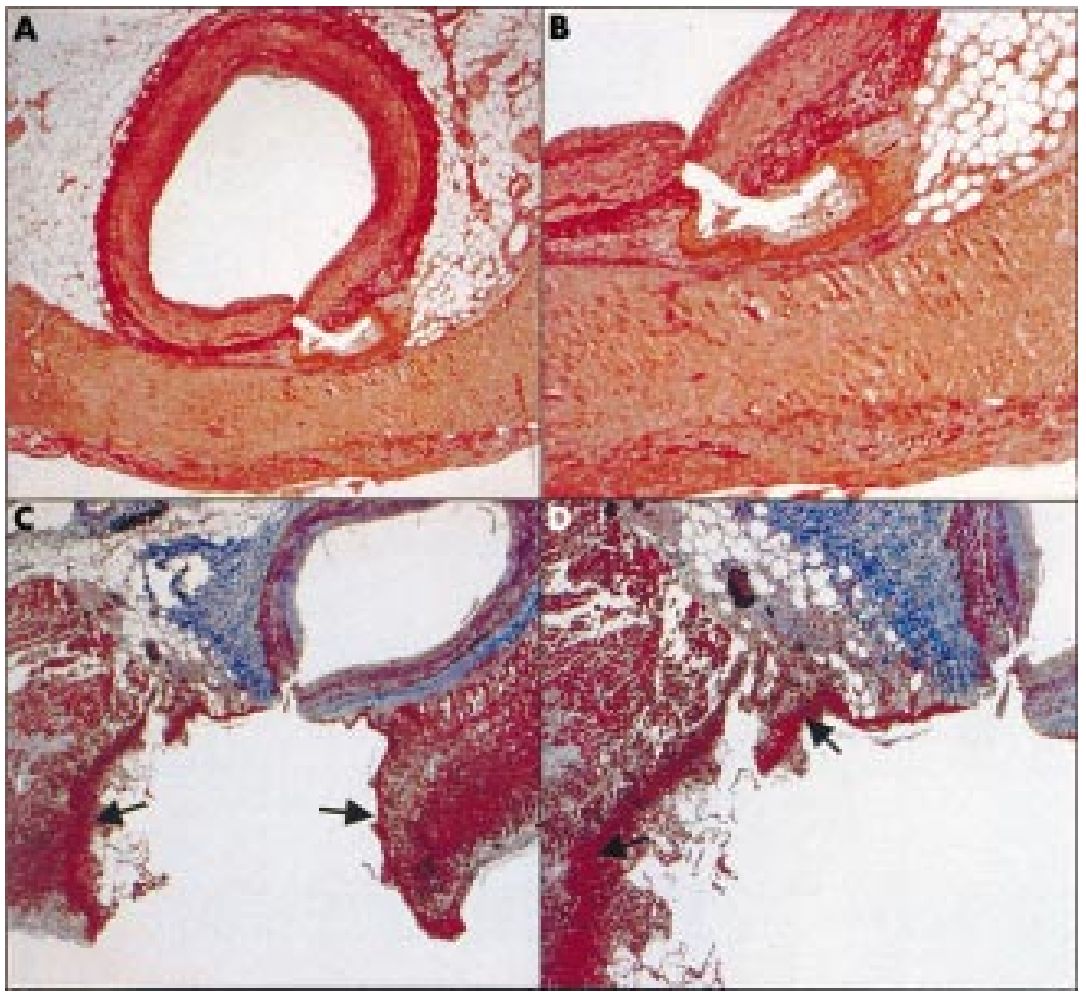

(A) Histological section of one of the two ruptured branches of the intermediate coronary artery. Note the sharp edges of the ruptured artery with formation of a pseudoaneurysm and a myocardial bridge that might have contributed to the atypical clinical presentation. Reactive fibrous pericarditis is also present (elastic van-Gieson stain). (B) A close-up view of (A). (C) Histologic section of the second ruptured intermediate branch with formation of a large pseudoaneurysm. (D) Close-up view of the site of rupture. Note the deposition of fibrin (in red) on the walls of the pseudoaneurysm (arrows) (trichrome stain Haidenhaim).

This is to our knowledge the first reported case of multiple coronary artery rupture following blunt chest trauma.

K Dimopoulos

A Angelini

R Mencarelli

G Thiene

cardpath@unipd.it 